Ann. Biol. anim. Bioch. Biophys., I974, 14 (I), I3I-I43.

\title{
ÉTUDE DE LA VARIATION \\ DE LA COMPOSITION AZOTÉE \\ DU MUSCLE PSOAS MAJOR \\ CHEZ TROIS RACES DE LAPINS ET LEURS CROISEMENTS RÉCIPROQUES
}

\author{
P. VIGNERON et J. NOUGUES \\ avec la collaboration technique de Odette Moulierac et L. Marger \\ Station de Physiologie animale, \\ École nationale supérieure agronomique, $I . N . R . A$, \\ Place Viala, \\ 34060 Montpellier Cedex
}

\section{RÉSUMÉ}

La variation de la composition azotée du psoas major a été étudiée chez des lapins abattus au poids vif de 2 ooo g, répartis en groupes représentant les races Commun gris, Néo-Zélandais, Blanc de Bouscat, et les produits de croisement des Néo-Zélandais avec les autres races. Pour la race Blanc de Bouscat, le poids du muscle étudié est significativement inférieur à celui des autres, au seuil $5 \mathrm{p}$. Ioo.

L'analyse a porté sur les caractères suivants :

$$
\begin{aligned}
& \mathrm{A}=\text { teneur en azote total du tissu frais } \times \text { Ioo; } \\
& \mathrm{B}=\text { azote } \mathrm{du} \text { stroma } / \mathrm{N} \text { total } \times \text { roo; } \\
& \mathrm{C}=\text { azote myofibrillaire } / \mathrm{N} \text { total } \times \text { Ioo; } \\
& \mathrm{D}=\mathrm{N} \text { sarcoplasmique } / \mathrm{N} \text { total } \times \text { roo. }
\end{aligned}
$$

Dans les deux sexes, il apparaît, intragroupe, une corrélation négative entre $B$ et $C, C$ et $D$, $B$ et $D$. L'analyse de variance révèle des différences entre sexes : pour certains groupes, les caractères $\mathrm{A}, \mathrm{B}, \mathrm{D}$ chez les mâles, $\mathrm{C}$ et $\mathrm{D}$ chez les femelles, sont significativement différents. Une analyse en composantes principales montre qu'il est possible de discriminer certains individus à l'intérieur des groupes, à l'aide d'une composante de "musculation " et d'une composante de " tendreté " qui expriment ensemble 75 p. Ioo de la variance totale. L'analyse canonique souligne que, chez les mâles, la discrimination de certains groupes peut se faire principalement par la variation de $\mathrm{D}$, chez les femelles par la variation de C. Cette analyse indique, pour les races pures, une différence entre Blancs de Bouscat d'une part, Néo-Zélandais et Communs d'autre part. Parmi les croisements, les groupes Néo-Zélandais $\times$ Blanc de Bouscat s'apparentent davantage aux Néo-Zélandais et sont parfois significativement différents des Blancs de Bouscat. De la même façon, les individus issus de croisements entre Néo-Zélandais et Communs ont davantage les caractères de cette dernière race. Il ressort donc de cette étude que la composition azotée d'un muscle est susceptible de présenter des variations d'origine génétique entre races, chez le Lapin. 


\section{INTRODUCTION}

Ia composition azotée du muscle squelettique présente des variations qui peuvent provenir du muscle étudié, de l'âge et du poids de l'animal, de son activité physique, de son origine génétique, ou de modifications d'ordre pathologique.

Au cours de la croissance, une évolution de la composition relative du muscle a été observée chez plusieurs espèces. Les résultats, obtenus à l'aide de diverses techniques, par Robinson (I952), Mizuno et al. (I966), Baril, et HERmann (I967), GuTmann et Syrovy (I967), Pot'ter et Hermann (I970) chez le Poulet, Gordon et al. (I966), chez le Rat, Perry et Hartshorne (I963) chez le Lapin, Dickerson et WidDowson (I960) chez le Porc et l'Homme, L,INK et al. (I970) chez les bovins, permettent de conclure à un enrichissement rapide du muscle en protéine myofibrillaire au cours de la période périnatale, une composition relative proche de celle de l'adulte semblant être atteinte très rapidement.

Dans le cadre d'un travail sur l'évolution postnatale de la composition musculaire du Lapin (BARON, VIGNERON, 1970), nous avons pu constater le même type d'évolution. Mais nous avons également relevé, pour certains individus ou certaines familles, des différences de composition d'une amplitude nettement supérieure à celle des erreurs expérimentales.

Nos animaux étant élevés dans des conditions uniformes, ce qui élimine l'activité physique comme facteur de variation possible (HELANDER, I96I), nous avons envisagé 1'hypothèse d'une origine génétique de ces différences. NouguEs (I972), étudiant l'évolution du nombre et des dimensions des fibres musculaires au cours de la croissance chez le Lapin, avait pu observer également une variabilité en rapport avec des facteurs génétiques.

Un petit nombre d'études des variations génétiques de la composition musculaire ont été effectuées dans la perspective d'une meilleure connaissance de l'évolution post-mortem de la viande (Mc LoughLIN, I968), ou au cours de travaux sur l'hypertrophie musculaire (BOCCARD, I968, AsHMORE et RoBINSON, I969, IODICE et al., I $972 \ldots)$. Une étude de ce type n'avait jamais été réalisée chez le Lapin, ce que nous avons entrepris de faire chez 3 races et leurs croisements, cependant que l'étude des variations du poids de certains muscles et des dimensions de leurs fibres était poursuivie parallèlement chez les mêmes individus (Nougues et al., I973).

\section{MATÉRIEL E'T MÉTHODES}

Nous avons entrepris d'étudier la composition protéique de muscles de lapins des deux sexes, abattus au poids vif de $2000 \mathrm{~g}$, rassemblés en groupes correspondant aux races Commun gris (I) Néo-Zélandais (2), Blanc de Bouscat (3), et à leurs croisements réciproques. Le plan expérimental est présenté au tableau I. Tous les croisements avec la race Blanc de Bouscat n'ont pu être étudiés, du fait de la disparition de reproducteurs ayant montré des difficultés d'adaptation 
à l'élevage sur grilles. Dans la nomenclature des produits de croisement, nous avons toujours présenté la race paternelle suivie de la race maternelle.

Notre étude a été réalisée sur le muscle psoas major, pour lequel nous avions eu l'occasion de constater certaines variations de composition. La méthode d'analyse chimique a déjà été exposée (BARON, VIGNERON, I970). Elle repose sur la solubilisation différentielle de fractions azotées à l'aide de milieux de force ionique différente (HELANDER, I957).

Outre la concentration du tissu frais en azote total ( $N$ total, variable $A$ ), cette méthode permet d'estimer 4 fractions, exprimées ici en p. Ioo de l'azote total :

Azote du stroma/ $\mathbf{N}$ total $\times$ roo $(\mathrm{N}$ stroma, Variable $\mathrm{B})$;

Azote myofibrillaire $/ \mathrm{N}$ total $\times$ Ioo ( $\mathrm{N}$ myo, Variable $\mathrm{C})$;

Azote sarcoplasmique $/ \mathrm{N}$ total $\times$ Ioo $(\mathrm{N}$ sarco, Variable $\mathrm{D})$;

Azote non protéique, fraction non retenue pour l'analyse statistique du fait des très faibles variations observées entre individus, quelle qu'en soit l'origine.

L'analyse statistique des résultats, réalisée sur ordinateur IBM I I 30 (Laboratoire de Biométrie, I. N. R. A., Champenoux), porte sur les variables A, B, C, D. Elle consiste en :

- une étude des corrélations entre caractères à l'intérieur de chaque groupe d'individus, et pour l'ensemble des groupes mâles ou femelles.

- une analyse de variance, a fin de comparer les fluctuations des caractères observés autour de la moyenne générale, et autour des moyennes des diverses races et croisements.

- une analyse en composantes principales (Rouvier, I966), qui permet d'étudier la discrimination des individus à l'intérieur de chaque groupe.

- une analyse canonique, dont le but est la discrimination des groupes entre eux.

\section{TABLEAU I}

Plan de croisement,

effectifs expévimentaux et poids moyens en $g$ des muscles psoas major prélevés (士 écart-type) pour chaque race ou croisement

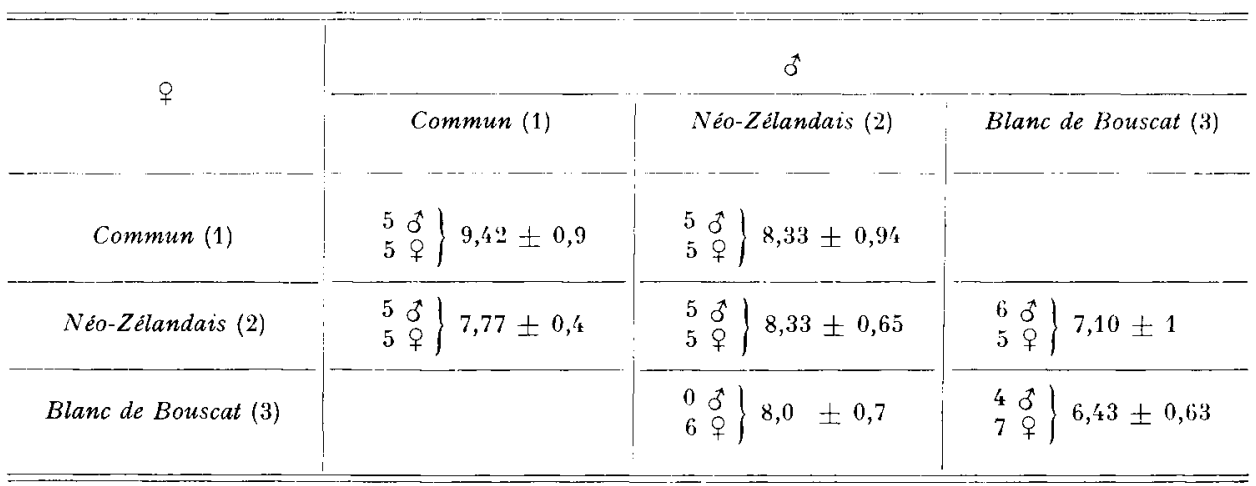

\section{RÉSULTATS}

Le poids moyen des muscles prélevés pour chaque race ou croisement est rapporté dans le tableau I. Il varie de $9,42 \mathrm{~g}$ pour la race I (Communs) à $6,43 \mathrm{~g}$ pour la race 3 (Blanc de Bouscat) qui, à cet égard, diffère significativement des autres groupes au seuil 5 p. Ioo. Pour l'ensemble il est de $7,84 \pm \mathrm{I}, \mathrm{I} 2 \mathrm{~g}$.

L'azote non protéique représente $4,66 \pm 0,06 \mathrm{mg} / \mathrm{g}$ de tissu frais. bleau 2.

Les moyennes et écart-types des autres variables sont présentés dans le ta- 
TABLEAU 2

Moyennes et écart-types des variables, pour chaque race ou croisement

\begin{tabular}{|c|c|c|c|c|c|c|c|c|c|}
\hline \multicolumn{2}{|c|}{ Race et croisement } & 1 & 2 & 3 & $2 \times 3$ & $3 \times 2$ & $1 \times 2$ & $2 \times 1$ & $\begin{array}{l}\text { Moyennes } \\
\text { générales }\end{array}$ \\
\hline \multirow{5}{*}{ Mâles } & Effectifs & 5 & 5 & 4 & & 6 & 5 & 5 & $(30)$ \\
\hline & N Total mg/g tissu frais & $\begin{array}{r}33,28 \\
0,41\end{array}$ & $\begin{array}{r}33,09 \\
1,30\end{array}$ & $\begin{array}{r}30,83 \\
1,84\end{array}$ & & $\begin{array}{r}32,86 \\
1,13\end{array}$ & $\begin{array}{r}33,30 \\
0,89\end{array}$ & $\begin{array}{r}32,82 \\
0,68\end{array}$ & 32,76 \\
\hline & $\mathrm{N}$ Stroma $/ \mathrm{N}$ total $\times 100$ & $\begin{array}{r}10,48 \\
2,48\end{array}$ & $\begin{array}{l}9,35 \\
3,64\end{array}$ & $\begin{array}{l}9,38 \\
0,86\end{array}$ & & $\begin{array}{l}8,50 \\
1,63\end{array}$ & $\begin{array}{l}6,97 \\
1,31\end{array}$ & $\begin{array}{l}5,64 \\
0,92\end{array}$ & 8,36 \\
\hline & $\mathrm{N}$ Myo $/ \mathrm{N}$ total $\times 100$ & $\begin{array}{r}50,65 \\
2,72\end{array}$ & $\begin{array}{r}52,03 \\
2,50\end{array}$ & $\begin{array}{r}54,53 \\
0,92\end{array}$ & & $\begin{array}{r}53,23 \\
2,77\end{array}$ & $\begin{array}{r}51,62 \\
2,71\end{array}$ & $\begin{array}{l}53 \\
1,87\end{array}$ & 52,47 \\
\hline & $\mathrm{N}$ Sarco $/ \mathrm{N}$ total $\times 100$ & $\begin{array}{r}24,68 \\
1,18\end{array}$ & $\begin{array}{r}24,79 \\
2,18\end{array}$ & $\begin{array}{r}21,72 \\
1,37\end{array}$ & & $\begin{array}{r}24,19 \\
2,03\end{array}$ & $\begin{array}{r}27,25 \\
4,06\end{array}$ & $\begin{array}{r}27,23 \\
2,29\end{array}$ & 23,06 \\
\hline \multirow{5}{*}{ l'emelles } & Effectifs & 5 & 5 & 7 & 6 & 4 & 5 & 5 & (37) \\
\hline & $\mathrm{N}$ Total $\mathrm{mg} / \mathrm{g}$ tissu frais & $\begin{array}{r}33,68 \\
1,30\end{array}$ & $\begin{array}{r}33,12 \\
0,64\end{array}$ & $\begin{array}{r}31,93 \\
1,24\end{array}$ & $\begin{array}{r}33,24 \\
0,95\end{array}$ & $\begin{array}{r}32,74 \\
2,70\end{array}$ & $\begin{array}{r}34,59 \\
1,24\end{array}$ & $\begin{array}{r}32,86 \\
0,48\end{array}$ & 33,11 \\
\hline & N Stroma $/ N$ total $\times 100$ & $\begin{array}{l}7,91 \\
1,72\end{array}$ & $\begin{array}{r}11,36 \\
4,31\end{array}$ & $\begin{array}{l}8,11 \\
1,35\end{array}$ & $\begin{array}{l}8,97 \\
3,78\end{array}$ & $\begin{array}{l}9,95 \\
0,65\end{array}$ & $\begin{array}{l}6,59 \\
1,86\end{array}$ & $\begin{array}{l}9,02 \\
3,07\end{array}$ & 8,78 \\
\hline & $\mathrm{N}$ Myo $/ \mathrm{N}$ total $\times 100$ & $\begin{array}{r}51,69 \\
1,33\end{array}$ & $\begin{array}{r}51,14 \\
3,87\end{array}$ & $\begin{array}{r}53,71 \\
1,97\end{array}$ & $\begin{array}{r}46,96 \\
3,01\end{array}$ & $\begin{array}{r}49,45 \\
2,06\end{array}$ & $\begin{array}{r}53,51 \\
2,23\end{array}$ & $\begin{array}{r}51,26 \\
3,32\end{array}$ & 51,17 \\
\hline & N Sarco $/ N$ total $\times 100$ & $\begin{array}{r}26,65 \\
0,85\end{array}$ & $\begin{array}{r}23,23 \\
1,50\end{array}$ & $\begin{array}{r}23,81 \\
1,74\end{array}$ & $\begin{array}{r}29,48 \\
1,63\end{array}$ & $\begin{array}{r}26,15 \\
2,53\end{array}$ & $\begin{array}{r}25,38 \\
2,21\end{array}$ & $\begin{array}{r}25,71 \\
3,43\end{array}$ & 25,76 \\
\hline \multirow{5}{*}{$\begin{array}{c}\text { Mâles } \\
+ \\
\text { Femelles }\end{array}$} & Effectifs & 10 & 10 & 11 & & 10 & 10 & 10 & (67) \\
\hline & N Total & $\begin{array}{r}33,48 \\
0,97\end{array}$ & $\begin{array}{r}33,10 \\
1,03\end{array}$ & $\begin{array}{r}31,38 \\
1,47\end{array}$ & & $\begin{array}{r}32,80 \\
1,88\end{array}$ & $\begin{array}{r}33,94 \\
1,08\end{array}$ & $\begin{array}{r}32,84 \\
0,59\end{array}$ & 32,96 \\
\hline & N Stroma/N total & $\begin{array}{l}9,20 \\
2,13\end{array}$ & $\begin{array}{r}10,35 \\
3,99\end{array}$ & $\begin{array}{l}8,74 \\
1,21\end{array}$ & & $\begin{array}{l}9,23 \\
1,35\end{array}$ & $\begin{array}{l}6,78 \\
1,61\end{array}$ & $\begin{array}{l}7,33 \\
2,27\end{array}$ & 8,59 \\
\hline & - N Myo/N total & $\begin{array}{r}41,17 \\
2,14\end{array}$ & $\begin{array}{r}51,59 \\
3,26\end{array}$ & $\begin{array}{r}54,12 \\
1,69\end{array}$ & & $\begin{array}{r}51,34 \\
2,53\end{array}$ & $\begin{array}{r}52,56 \\
2,49\end{array}$ & $\begin{array}{r}52,13 \\
2,69\end{array}$ & 51,75 \\
\hline & N Sarco $/ N$ total & $\begin{array}{r}25,67 \\
1,03\end{array}$ & $\begin{array}{r}24,01 \\
1,87\end{array}$ & $\begin{array}{r}22,76 \\
1,62\end{array}$ & & $\begin{array}{r}25,17 \\
2,23\end{array}$ & $\begin{array}{r}26,32 \\
3,27\end{array}$ & $\begin{array}{r}26,47 \\
2,91\end{array}$ & 25,44 \\
\hline
\end{tabular}

\section{I. - Corrélations entre caractères}

Les matrices de corrélations entre caractères (tab1. $3 c$ ) calculées intragroupes pour l'ensemble des groupes mâles ou femelles font apparaître une corrélation négative entre azote du stroma et azote myofibrillaire $(-0,474,-0,685)$, azote myofibrillaire et azote sarcoplasmique $(-0,667,-0,4 \mathrm{I} 7)$, ainsi qu'entre azote sarcoplasmique et azote du stroma (- $0,3 \mathrm{II},-0,326)$. 


\section{TABLEAU 3}

Corrélations ( $\times$ Iooo) entre caractères intragroupes

a. Par groupe d'individus mâles

\begin{tabular}{|c|c|c|c|c|c|c|}
\hline \multirow[b]{2}{*}{ Caractères } & \multicolumn{6}{|c|}{ Groupes } \\
\hline & $\begin{array}{c}1 \\
\text { (Communs) }\end{array}$ & $\begin{array}{c}2 \\
\text { (Nêo-Zélandais) }\end{array}$ & $\begin{array}{c}3 \\
\text { (Bouscat) }\end{array}$ & $3 \times 2$ & $1 \times 2$ & $2 \times 1$ \\
\hline$A-B$ & 57 & 403 & -176 & 56 & 491 & 36 \\
\hline$A-C$ & 208 & -497 & -539 & 106 & 844 & -206 \\
\hline$A-D$ & -949 & -103 & 645 & -86 & -787 & - \\
\hline B-C & -938 & -775 & -117 & -659 & 826 & 一 \\
\hline$B-D$ & 222 & -552 & -644 & 205 & -896 & -497 \\
\hline$C-D$ & -468 & -86 & -671 & -864 & -989 & -814 \\
\hline
\end{tabular}

b. Par groupe d'individus femelles

\begin{tabular}{|c|c|c|c|c|c|c|c|}
\hline \multirow{2}{*}{ Caractères } & \multicolumn{7}{|c|}{ Groupes } \\
\hline & 1 & 2 & 3 & $3 \times 2$ & $2 \times 3$ & $1 \times 2$ & $2 \times 1$ \\
\hline$A-B$ & 511 & 260 & 47 & 303 & 121 & -754 & -182 \\
\hline$A-C$ & -619 & -371 & 376 & -995 & 148 & 718 & -157 \\
\hline$A-D$ & $-\quad 44$ & 687 & -188 & 999 & -227 & -154 & 442 \\
\hline B-C & -912 & -966 & -470 & 386 & -793 & -607 & -281 \\
\hline B-D & -374 & -150 & -115 & 280 & -676 & -164 & -600 \\
\hline$C-D$ & -20 & -52 & -746 & -992 & 103 & -675 & -577 \\
\hline & & & & & & & \\
\hline
\end{tabular}

c. Pour l'ensemble des groupes

\begin{tabular}{|c|c|c|c|c|c|c|c|c|c|}
\hline \multicolumn{5}{|c|}{ Mâles } & \multicolumn{5}{|c|}{ Femelles } \\
\hline Caractères & A & $\mathrm{B}$ & $\mathrm{C}$ & $\mathrm{D}$ & Caractères & A & $\mathrm{B}$ & $\mathrm{C}$ & $\mathrm{D}$ \\
\hline $\begin{array}{l}\mathrm{A} \\
\mathrm{B} \\
\mathrm{C} \\
\mathrm{D}\end{array}$ & $\begin{array}{r}1000 \\
181 \\
7 \\
-165\end{array}$ & $\begin{array}{r}1000 \\
-474 \\
-311\end{array}$ & $\begin{array}{r}1000 \\
-667\end{array}$ & 1000 & $\begin{array}{l}\text { A } \\
\text { B } \\
\text { C } \\
\text { D }\end{array}$ & $\begin{array}{r}1000 \\
-34 \\
-95 \\
244\end{array}$ & $\begin{array}{r}1000 \\
-685 \\
-326\end{array}$ & $\begin{array}{r}1000 \\
-417\end{array}$ & 1000 \\
\hline \multicolumn{5}{|c|}{$\begin{array}{l}\mathrm{A}=\mathrm{N} \text { Total mg/g tissu frais; } \\
\mathrm{B}=\mathrm{N} \text { Stroma } / \mathrm{N} \text { Total } ;\end{array}$} & \multicolumn{3}{|c|}{$\begin{array}{l}\mathrm{C}=\mathrm{N} \text { Myo/N Total } \\
\mathrm{D}=\mathrm{N} \text { Sarco/N Total. }\end{array}$} & & \\
\hline
\end{tabular}


Si l'on considère maintenant pour un sexe donné, les groupes pris séparément (tabl. $3 a$ et $3 b$ ), on observe que les variables $\mathrm{C}$ et $\mathrm{D}$ ( $\mathrm{N}$ myo, $\mathrm{N}$ sarco) sont peu corrélées entre elles chez les animaux Communs et Néo-Zélandais de race pure. Parmi les croisements, il apparaît que le groupe $\mathrm{I} \times 2$ (mâles Communs $\times$ femelles $N e ́ o-$ Zélandaises) se différencie des autres, tant chez les mâles que chez les femelles, par la valeur positive élevée des corrélations entre les caractères $\mathrm{A}$ et $\mathrm{C}$ ( $\mathrm{N}$ total, $\mathrm{N}$ myo).

$A$ ces exceptions près, on peut admettre que les corrélations entre caractères pour les 7 groupes sont assez homogènes et rendent possible l'analyse discriminante.

\section{2. - Analyse de variance}

Les moyennes et écarts-types de nos résultats sont présentés dans le tableau 2.

$L$ 'analyse de variance a fait apparaître certaines différences dans les résultats des deux sexes (tabl. 4). Chez les mâles, les caractères $\mathrm{A}, \mathrm{B}, \mathrm{D}$ ( $\mathrm{N}$ total, $\mathrm{N}$ stroma, $\mathrm{N}$ sarco) sont significativement différents, au seuil $5 \mathrm{p}$. Ioo, pour certains groupes. Chez les femelles, $\mathrm{C}$ et $\mathrm{D}$ ( $\mathrm{N}$ myo, $\mathrm{N}$ sarco) sont significativement différents au seuil I p. Ioo.

\section{TABLEAU 4}

Résultats de l'analyse de variance (valeurs de F) pour chaque sexe

\begin{tabular}{|c|c|c|}
\hline \multirow{2}{*}{ Variables } & \multicolumn{2}{|c|}{ Sexe $(n=)$} \\
\hline & $\begin{array}{l}\text { Mâles } \\
(30)\end{array}$ & $\begin{array}{c}\text { Femelles } \\
\text { (37) }\end{array}$ \\
\hline A N Total & $3,03^{*}$ & NS \\
\hline $\mathrm{B} \frac{\mathrm{N} \text { Stroma }}{\mathrm{N} \text { Total }}$ & $3,63^{*}$ & NS \\
\hline$C \frac{\mathrm{N} \text { Total }}{\mathrm{N} \text { Myo }}$ & NS & ${ }^{4}, 52 * *$ \\
\hline D $\frac{\text { N Sarco }}{\mathrm{N} \text { Total }}$ & $3,40^{*}$ & $5,70^{* *}$ \\
\hline $\begin{array}{l}\text { NS Non significa } \\
\text { Seuils de signific }\end{array}$ & 0,$05 ; * * 0,01$ & \\
\hline
\end{tabular}

\section{3. - Analyses en composantes principales}

Les vecteurs propres, ou composantes principales $\mathrm{Y}_{1}, \mathrm{Y}_{2}, \mathrm{Y}_{3}, \mathrm{Y}_{4}$ sont des fonctions linéaires en rapport avec les variables initiales, telles que :

$$
\mathrm{Y}=a \mathrm{~A}+b \mathrm{~B}+c \mathrm{C}+d \mathrm{D} \text {. }
$$

Ils forment un système d'axes non corrélés entre eux, ce qui permet de les étudier séparément et de discriminer au mieux les individus d'un même groupe.

Chez les mâles et chez les femelles, $Y_{1}$ et $Y_{2}$ rendent compte à eux seuls des trois 
quarts de la variance totale (tabl. 5). I a discrimination des individus s'en trouve donc simplifiée et peut se faire uniquement à partir de ces deux vecteurs. D'autre part, le tableau des corrélations entre vecteurs propres et variables de départ (tabl. 5) met en évidence des différences entre sexes.

Chez les mâles, $\mathrm{Y}_{1}=-0, \mathrm{II} 2 \mathrm{~A}+0, \mathrm{I} 83 \mathrm{~B}-0,735 \mathrm{C}+0,643 \mathrm{D}$.

Chez les femelles, $\mathrm{Y}_{1}=0,20 \mathrm{~A}+0,6 \mathrm{I} \mathrm{B}-0,735 \mathrm{C}+0,2 \mathrm{I} 7 \mathrm{D}$.

$\mathrm{Y}_{1}$ traduit donc une opposition dans la variation de $\mathrm{C}$ et de $\mathrm{D}$ ( $\mathrm{N}$ myo, $\mathrm{N}$ sarco) chez les mâles, de B et de C ( $\mathrm{N}$ stroma, $\mathrm{N}$ myo) chez les femelles.

Chez les mâles, $\mathrm{Y}_{2}=0,477 \mathrm{~A}+0,754 \mathrm{~B}-0,227 \mathrm{C}-0,39 \mathrm{D}$.

Chez les femelles, $\mathrm{Y}_{2}=0,444 \mathrm{~A}-0,485 \mathrm{~B}-0,006 \mathrm{C}+0,75 \mathrm{D}$. Dans les deux cas $\mathrm{Y}_{2}$ représente l'opposition dans la variation de $\mathrm{B}$ et de $\mathrm{D}$, mais tient compte également de $\mathrm{A}$ ( $\mathrm{N}$ total).

$\mathrm{Y}_{3}$ représente presque exclusivement le caractère $A(N$ total) quei que soit le sexe. Il rend compte à lui seul de $22 \mathrm{p}$. Ioo de la somme des variances des caractères, et permet une certaine discrimination des animaux intragroupe.

\section{TABLEAU 5}

Corrélations entre variables expérimentales et composantes principales, et pourcentage de la somme des variances des variables exprimé par chaque composante principale

\begin{tabular}{|c|c|c|c|c|c|c|c|c|}
\hline \multirow{2}{*}{$\begin{array}{l}\text { Composante principale } \\
\text { Sexe des animaux }\end{array}$} & \multicolumn{2}{|c|}{$Y_{1}$} & \multicolumn{2}{|c|}{$Y_{2}$} & \multicolumn{2}{|c|}{$Y_{3}$} & \multicolumn{2}{|c|}{$Y_{4}$} \\
\hline & $\hat{o}$ & q & 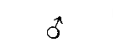 & q & 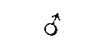 & q & $\hat{\jmath}$ & 우 \\
\hline$-\quad---$ & - & & & -- . & & & & \\
\hline A (N Total) & $-0,146$ & 0,263 & 0,568 & 0,523 & 0,81 & 0,81 & 0,0005 & 0,012 \\
\hline $\mathrm{B}$ (N Stroma/N total) & 0,238 & 0,801 & 0,898 & $-0,572$ & $-0,364$ & 0,152 & 0,058 & 0,087 \\
\hline $\mathrm{C}(\mathrm{N} \mathrm{Myo} / \mathrm{N}$ Total $)$ & $-0,959$ & $-0,963$ & $-0,269$ & $-0,071$ & 0,025 & $0,2 /$ & 0,075 & 0,09 \\
\hline D (N Sarco/N Total) & 0,839 & 0,285 & $-0,46$. & 0,885 & 0,272 & $-0,36$ & 0,07 & 0,07 \\
\hline$\%$ de la variance totale & 42,6 & 43 & 35,45 & $3 y_{t}^{\prime}, 73$ & 21,6 & 21,7 & 0,35 & 0,52 \\
\hline
\end{tabular}

\section{4. - Analyse canonique}

De même que les composantes principales, les variables canoniques $Z i$ sont des fonctions, combinaisons linéaires de caractères qui peuvent être soit, comme ici, les variables initiales, soit les composantes principales (Rouviek, I966, DAvAINE et al., I969). Ces fonctions permettent de discriminer les moyennes des populations.

Les deux premières variables canoniques rendent compte à elles seules de plus de $75 \mathrm{p}$. Ioo de la somme des variances intergroupes des variables initiales chez les mâles, les femelles, ou l'ensemble des groupes mâles et femelles. Un test de $\mathrm{X}_{2}$ montre que seul $Z_{1}$ peut permettre de discriminer certains groupes de mâles, alors que pour les femelles ou l'ensemble des groupes mâles et femelles, $Z_{1}$ et $Z_{2}$ le peuvent.

D'après la 'valeur des coefficients des variables dans $Z_{1}$ et $Z_{2}$, et celle des corrélations entre ces vecteurs et les variables initiales, il ressort que $Z_{1}$ représente surtout la variation de $\mathrm{A}$ ( $\mathrm{N}$ total) et de $\mathrm{D}$ ( $\mathrm{N}$ sarco) chez les mâles, de la variable $\mathrm{C}$ ( $\mathrm{N}$ myo) chez les femelles, et pour l'ensemble des groupes mâles et femelles de l'opposition 
de la variation de $C$ et de $D . Z_{2}$ est chez les femelles un vecteur composite, surtout corrélé aux variables A ( $\mathrm{N}$ total) et $\mathrm{B}$ ( $\mathrm{N}$ stroma). Pour l'ensemble des groupes, $Z_{2}$ rend bien compte de l'opposition de la variation de $\mathrm{B}$ et de $\mathrm{C}$.

Nous avons représenté sur la figure I, l'intersection des vecteurs canoniques $Z_{1}$ et $Z_{2}$, déterminés pour l'ensemble des groupes, avec le plan des variables $C$ et $D(N$ myo, $\mathrm{N}$ sarco), le mieux susceptibles de discriminer certains groupes. Dans ce plan sont également portées les valeurs moyennes de chacun des groupes de mâles ou de femelles. Cette figure résume bien les tendances générales de l'analyse canonique.

On voit que chez les mâles, les groupes I $\times 2$ et $2 \times$ I sont séparés des autres $\operatorname{par} Z_{1}$ du fait des valeurs de $\mathrm{D}\left(\mathrm{N}\right.$ sarco). Chez les femelles, $Z_{1}$ isole les groupes 3,2 , $3 \times 2$ et $Z_{2}$, les groupes $3,2, I \times 2$, les variables $C$ ou $D$ contribuant chacune à établir les différences.

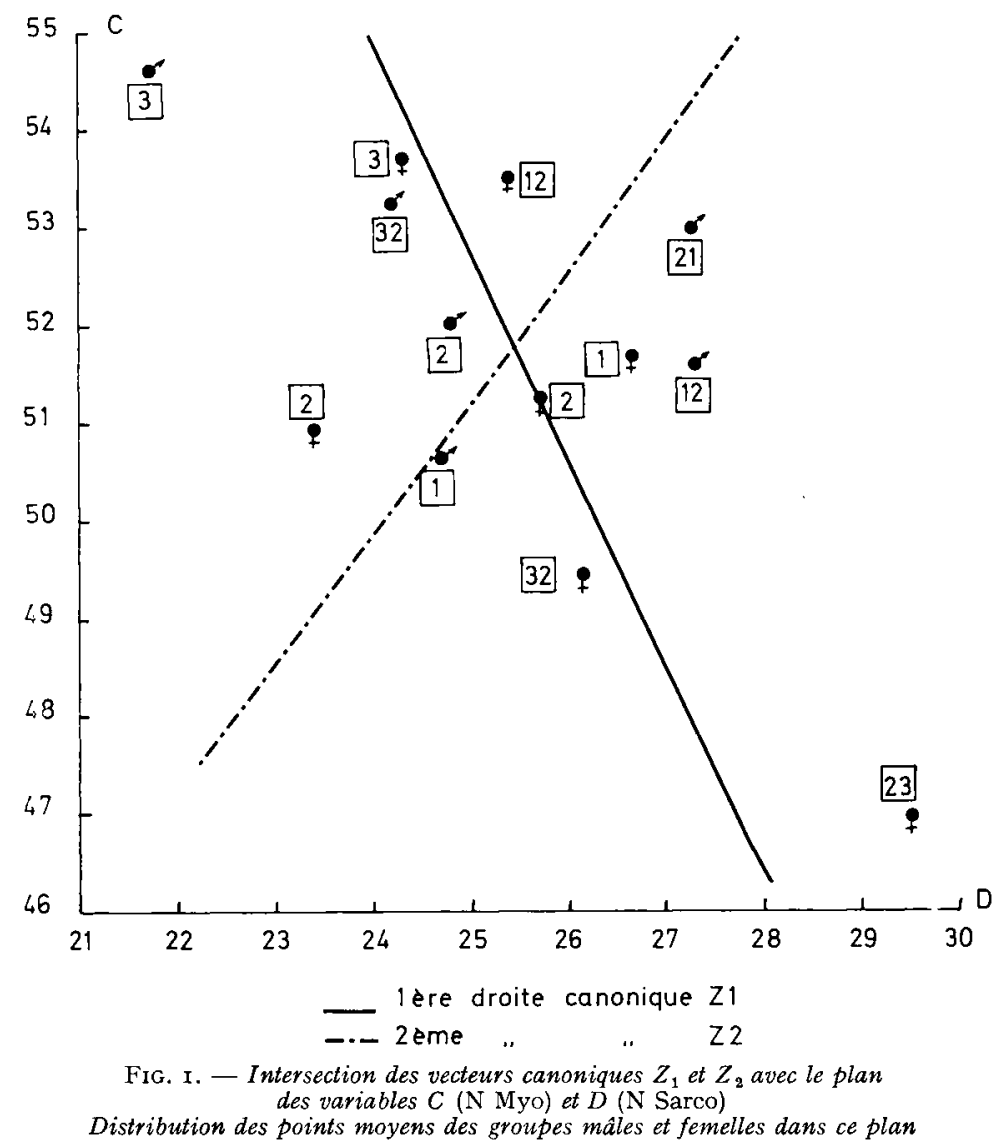

Les valeurs significatives de $\mathrm{F}$ pour les distances généralisées de Mahalanobis, calculées entre les valeurs moyennes des individus des différents groupes de mâles par comparaison aux groupes de femelles sont portées sur le tableau $6 a$. On trouve ces valeurs, calculées entre groupes de mâles, ou entre groupes de femelles, dans le tableau $6 b$. Elles mettent en évidence des différences significatives entre certains 


\section{TABLEAU $6 a$}

Valeurs de $F$ correspondant aux distances généralisées de Mahalanobis calculées entre les valeurs moyennes des individus des différents groupes de mâles et de femelles

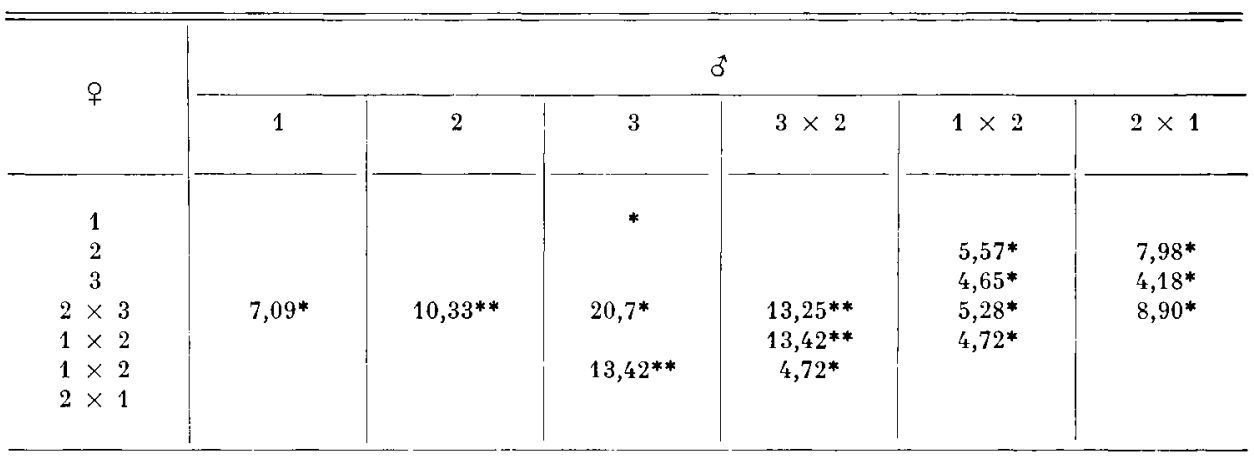

Seules les valeurs significatives ont été portées sur le tableau.

Seuils de signification : * 5 p. $100 ;{ }^{* *} 1$ p. 100.

TABLEAU $6 \quad b$

Valeurs de $F$ correspondant aux distances généralisées

de Mahalanobis calculées entre les valeurs moyennes

des individus des différents groupes de mâles (au-dessus de la diagonale), ou de femelles (en dessous de la diagonale)

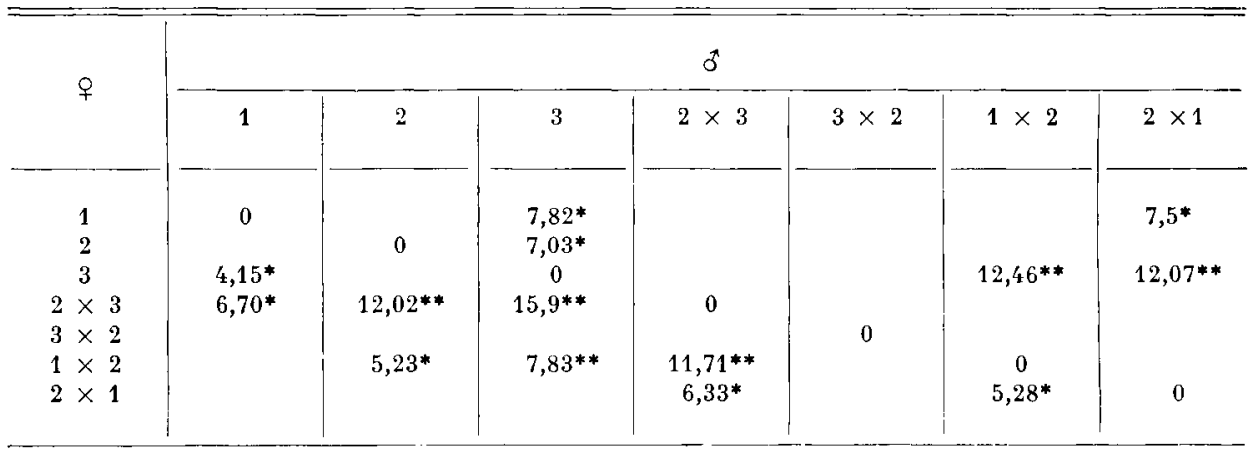

Seules les valeurs significatives ont été portées sur le tableau.

Seuils de signification : ${ }^{*} 5$ p. $100 ;{ }^{* *} 1$ p. 100. 
groupes, et précisent les impressions tirées de la lecture de la figure r. Les tendances suivantes s'en dégagent :

Parmi les animaux de race pure, les Blanc de Bouscat se différencient des Communs et des Néo-Zélandais, principalement par le caractère $\mathrm{C}$ ( $\mathrm{N}$ myo).

Les animaux issus de croisements entre Néo-Zélandais (2) et Communs (I) sont plus proches des Communs, le caractère $\mathrm{D}(\mathrm{N}$ sarco) faisant la différence. Enfin, certains croisements entre Néo-Zélandais (2) et Blanc de Bouscat (3) diffèrent notablement de ceux-ci par les caractères $\mathrm{D}(\mathrm{N}$ sarco) et surtout $\mathrm{C}(\mathrm{N}$ myo). Le groupe $2 \times 3$ (femelles) est d'ailleurs remarquablement différent de la plupart des autres.

\section{DISCUSSION}

La différence significative de poids constatée entre le psoas major des Blanc de Bouscat et celui des autres groupes pose le problème de l'état de maturité des animaux étudiés. Néo-Zélandais et Communs atteignent, adultes, un poids vif du même ordre de grandeur alors que les Blanc de Bouscat ont un format nettement plus important. Ceux-ci sont donc, au poids vif de $2000 \mathrm{~g}$, relativement plus immatures que les autres et présentent la plus faible teneur en azote total. Cependant, les proportions relatives des diverses catégories de protéines musculaires évoluent très rapidement après la naissance et atteignent des valeurs proches de celles de l'adulte avant le quinzième jour chez le Lapin (BARON, Vigneron, I970, Vigneron, I973). Dans ces conditions, il ne semble pas que ces différences de développement aient pu sensiblement modifier notre analyse.

L'étude des matrices de corrélations entre caractères intrapopulations fait ressortir 3 corrélations négatives. Les deux premières, entre $\mathrm{B}$ et $\mathrm{C}$ ( $\mathrm{N}$ stroma, $\mathrm{N}$ myo) et entre $\mathrm{C}$ et $\mathrm{D}$ ( $\mathrm{N}$ myo, $\mathrm{N}$ sarco) pourraient s'expliquer par des différences dans la nature ou les dimensions des fibres musculaires. C'est ainsi que chez les bovins " culards ", comparés à des individus " normaux ", on observe une proportion plus importante de fibres à métabolisme anaérobie, de plus grand diamètre, avec diminution de la teneur en collagène, constituant principal du stroma (ASHMORE et RoBInson, I969). D'autre part, la proportion inverse N myo, N sarco, c'est-à-dire entre protéines contractiles et équipement enzymatique apparaît quand on compare la composition de muscles " rouges " et "blancs" : ceux-ci sont moins riches en azote myofibrillaire, plus riches en azote sarcoplasmique (MEYER et WEBER, I933). Enfin, la faible corrélation négative entre $B$ et $D(N$ stroma, $N$ sarco) pourrait provenir de différences de soiubilité du collagène, entre individus ou entre races.

Les différences entre sexes, établies par l'analyse de variance, ont une influence certaine dans la suite de l'analyse. Ainsi, dans l'analyse en composantes principales, la discrimination entre individus dans les groupes de mâles est faite pour $\mathrm{Y}_{1}$ par la variation relative de $\mathrm{C}$ et de $\mathrm{D}$ ( $\mathrm{N}$ myo, $\mathrm{N}$ sarco), et chez les femelles par la variation relative de $\mathrm{B}$ et de $\mathrm{C}$ ( $\mathrm{N}$ stroma, $\mathrm{N}$ myo). $\mathrm{Y}_{1}$ peut donc être considérée dans l'un et l'autre cas comme une composante de "musculation " puisqu'elle tient compte de l'opposition de la variation de l'azote myofibrillaire et d'un autre caractère. Par contre, des différences entre sexes n'apparaissent ni pour $Y_{2}$, composante de " ten- 
dreté ", qui représente les variations opposées de B (N stroma, c'est-à-dire essentiellement le conjonctif) et de $\mathrm{D}$ ( $\mathrm{N}$ sarco, protéines hydrosolubles), ni pour $\mathrm{Y}_{3}$, très fortement corrélée à l'azote total chez les mâles comme chez les femelles (tab1. 5).

Ces différences entre sexes réapparaissent au cours de l'analyse canonique. Chez les femelles, comme pour l'analyse en composantes principales, ce sont encore les variables $\mathrm{B}$ et surtout $\mathrm{C}$ ( $\mathrm{N}$ stroma, $\mathrm{N}$ myo) qui permettent la meilleure discrimination des groupes, alors que chez les mâles, ce sont surtout $\mathrm{A}$ ( $\mathrm{N}$ total) et, principalement, $\mathrm{D}(\mathrm{N}$ sarco). Chez ceux-ci, ce n'est donc pas exactement à partir des mêmes caractères que se fait la meilleure discrimination des individus d'une part, des groupes d'autre part.

Il apparaît ainsi que la composition d'un muscle peut présenter des variations, notamment dans les proportions relatives de l'azote myofibrillaire et sarcoplasmique, permettant de différencier certains individus et certaines races comme c'est ici le cas pour la race Blanc de Bouscat. De plus, mais à un moindre degré, le caractère seul, peut permettre une certaine discrimination intragroupe, et dans certains cas, entre groupes. En outre, nous constatons que les valeurs des variables, pour les produits de croisement, ne représentent généralement pas la moyenne de celles des races parentales. En particulier, les croisements Néo-Zélandais $\times$ Blanc de Bouscat s'apparentent davantage aux Néo-Zélandais et sont parfois même significativement différents des Blancs de Bouscat.

Des différences génétiques de composition musculaire ont cependant été rarement rapportées. Mc Loughlin (I968) n'avait pas observé de différences d'extractibilité des protéines myofibrillaires et sarcoplasmiques entre deux souches de porcs, alros que BocCARD (I968) constate, entre muscles de porcs normaux ou hypertrophiques, une différence hautement significative de la teneur en collagène, mesurée par la teneur en hydroxyproline. Ashmore et RoBInson (I969) ne relèvent pas de différences dans la teneur en ADN et en protéines de deux muscles, le semi tendinosus et le biceps brachii, de bovins normaux ou hypertrophiques (culards). Mais LAWRIE (I96r) donne des valeurs nettement différentes pour les fractions azotées de muscles de bovins de plusieurs origines.

Il n'y a donc pas concordance entre les travaux réalisés sur des espèces ou des races différentes. Chez le Lapin, nos résultats, acquis sur des lots peu importants d'animaux, sont en faveur d'une variation génétique entre races de la composition de certains muscles. Nous nous proposons donc de poursuivre un travail de même type sur un plus grand effectif en tenant compte des rapports qui existent entre la composition azotée et les proportions des divers types de fibres qu'ils renferment.

Rę̧u pour publication en juillet 1973.

\section{REMERCIEMENTS}

Nous remercions tout particulièrement M. R. RouvieR (laboratoire de Méthodologie génétique, I. N. R. A., Toulouse) ainsi que Mlle Anne-Marie Rous (Station de Physiologie animale, I. N. R. A., Montpellier) et M. C. Millier (Station de Biométrie, C. N. R. F., Nancy) pour les conseils qu'ils ont bien voulu nous apporter.

Ce travail a été effectué avec le concours financier de la Délégation Générale à la Recherche Scientifique et Technique (Contrat na 7 I 7 3129).

Annales de Biologie animale. - 1974. 


\section{SUMMARY}

\section{STUDY OF VARIATION IN NITROGEN COMPOSITION OF THE PSOAS MAJOR MUSCLE IN THREE BREEDS OF RABBITS AND THEIR RFCIPROCAL, CROSSES}

Variation in nitrogen composition of the psoas major was studied in rabbit slaughtered at $2000 \mathrm{~g}$ body weight, and divided into groups representing the Common grey, New Zealand and Blanc de Bouscat breeds and crosses of New Zealand with the other breeds. Muscle weight in the Blanc de Bouscat is significantly lower than that of other breeds (threshold : 5 p. Ioo).

Analysis included the following characters :
$A=$ amount of total nitrogen in fresh tissue $\times 100 ;$
$\mathrm{B}=$ stroma nitrogen/total $\mathrm{N} \times 100 ;$
$\mathrm{C}=$ myofibrillar nitrogen/total $\mathrm{N} \times 100$
$\mathrm{D}=\operatorname{sarcoplasm~} \mathrm{N} /$ total $\mathrm{N} \times$ гоo.

In the two sexes, there is negative intragroup correlation between $\mathrm{B}$ and $\mathrm{C}, \mathrm{C}$ and $\mathrm{D}, \mathrm{B}$ and $\mathrm{D}$. Analysis of variance shows differences between sexes : for some groups, the characters A, B, D in males ; $C$ and $D$ in females are significant differences. Analysis of the main components shows that some individuals within groups may be distinguished using " musculation " and " tenderness" components, which together express $75 \mathrm{p}$. I 100 of total variance. Canonic analysis emphasizes that in males some groups may be determined mainly by the variation of $D$, in females by the variation of $\mathrm{C}$. This analysis indicates that for pure breeds, there is a difference between Blanc de Bouscat on the one hand, and New Zealand and Common on the other. Among the crosses, New Zealand $\times$ Blanc de Bouscat are more closely related to New Zealand and are sometimes significantly different from Blanc de Bouscat. In the same way, crosses between New Zealand and Common have more of the characters of the latter breed. This study shows that muscle nitrogen composition in rabbit may present variations between breeds which are of genetic origin.

\section{RÉFÉRENCES BIBLIOGRAPHIQUES}

Ashmore C. R., Robinson D. W., I969. Hereditary muscular hypertrophy in the bovine. I. Histological and Biochemical Characterization. Proc. Soc. exp. Biol. Med., 132, 548-554.

Baril E. F., HerrmanN H., I967. Studies of muscle development. II. Immunological and enzymatic properties and accumulation of chromatographically homogeneous myosin of the leg musculature of the developing chick. Devl. Biol., 15, 318-333.

Baron R., Vigneron P., i97o. Hétérogonie biochimique des composants azotés du grand psoas au cours de la croissance postnatale du Lapin. Ann. Biol. anim. Bioch. Biophys., 10, 223-238.

Boccard R., 1968. Variation de la teneur en hydroxyproline de muscles de porcs Large White et Piétrain. Ann. Zootech., 17, 7I-75.

Davaine P., Rouvier R., Ricard F. H., I969. Relations entre les expressions de la variabilité morphologique intra et inter familles chez le Poulet. Biometr. Praxim., 10, I-2I.

Dickerson J. W. T., Widdowson E. M., I960. Chemical changes in skeletal muscle during development. Biochem. J., 74, 247-257.

Gordon E. E., Kowalski K., Fritts M., I966. Muscle proteins and DNA in rat quadriceps during growth. Am.J. Physiol., 210, 1033-1039.

Gutmann E., Syrovy I., I967. Metabolic differentiation of the anterior and posterior latissimus dorsi of the chicken during development. Physiol. Bohemoslov., 16, 232-243.

Helander E., 1957. On quantitative muscle protein determination. Acta physiol. scand., Suppl., 141, 9-95.

HeLANDer E., I96r. Influence of Exercise and restricted activity on the protein composition of skeletal muscle. Biochem. J., 78, 478-482.

Iodice A. A., Chin J., Perker S., Weinstock I. M., ig72. Cathepsins A, B, C, D and autolysis during development of breast muscle of normal and dystrophic chickens. Archs. Biochem. Biophys., 152, I $66-$ I 74 . 
LAwrie R. A., I961. Systematic analytical differences between psoas major and longissimus dorsi muscles of cattle. Br. J. Nutr., 15, 453-456.

Link B. A., Cassens R. G., Kauffman R. G., Bray R. W., i97o. Changes in the solubility of bovine muscle proteins during growth. J. Anim. Sci., 30, ro-I4.

Mcloughlin J. V., I 968 . Sarcoplasmic and myofibrillar protein in skeletal muscle of two breeds of Pig. J. Food. Sci., 33, 383-385.

Meyer K. H., Weber H. H., I933. Biochem. $Z$. 266, I37. Cité par Helander, I957.

Mizuno T., Hikami Y., Sumita K., Ig66. The accumulation of nucleic acid and protein in the skeletal muscle of chicks during embryonic and post-embryonic development. Jap. J. Zootech. Sci., 37, $471-477$.

Nougues J., I972. Étude de l'évolution du nombre des fibres musculaires au cours de la croissance postnatale du muscle chez le Lapin. C. R. Soc. Biol., 166, I65-172.

Nougues J., Rous A. M., Vigneron P., 1973. Étude de la variation du nombre et des dimensions des fibres musculaires chez trois races de lapins et leurs croisements réciproques. Ann. Biol. anim. Bioch. Biophys. (Sous presse).

Perry S. V., Hartshorne D. J., ig63 in Gutmann E., et Hnik P., Effect of use and disuse on neuromuscular functions. Elsevier : Amsterdam. $49 \mathrm{I}-498$.

Potter J. D., Herrmann H., I97o. Studies of muscle development. VI. Identification of tropomyosin and troponin and quantitation of tropomyosin in embryonic and mature chick leg muscle. Archs. Biochem. Biophys., 141, 271-277.

Rosinson D. S., I952. Changes in the protein composition of chick muscle during development. Biochem. J., 52, 62I-628.

Rouvier R., I966. L'analyse en composantes principales : son utilisation en génétique et ses rapports avec l'analyse discriminatoire. Biometrics., 22, 343-357.

Vigneron P., I973. Évolution de la composition azotée des muscles psoas major et longissimus dorsi, de la naissance à 3 semaines chez le Lapin. Ann. Biol. anim. Bioch. Biophys. (Sous presse). 